\title{
Educación Médica sobre alcohol: perspectiva de futuro*
}

\author{
Rodríguez-MARTos, A. \\ Pla d'Acció sobre Drogues . Institut Municipal de Salut Pública. Ajuntament de Barcelona \\ Enviar correspondencia a: \\ Pla d'Acció sobre Drogues.PI. Lesseps, 1.08023 - Barcelona ·email: amartos@imsb.bcn.es
}

\section{RESUMEN}

El objetivo de este trabajo es revisar la situación actual de la formación médica en Europa y presentar algunas propuestas para el futuro. Para ello se realizó una amplia revisión bibliográfica y se contactó con profesionales y universidades de toda la región europea.

De la información recogida, se desprende que la formación de los profesionales europeos todavía tiene muchas lagunas.

Se propone un plan de formación básico destinado a cubrir los 3 niveles de la carrera médica y se sugieren componentes educativos clave (conocimientos, habilidades y actitudes) estrategias de formación básicas destinadas sobre todo a capacitar a los médicos de atención primaria para la identificación precoz e intervención breve. Los objetivos del aprendizaje son: conocer los hechos, identificar los problemas de alcohol, distinguir a los pacientes que pueden beneficiarse de una intervención breve y adquirir habilidades para aplicarla. El objetivo final sería desarrollar un compromiso terapéutico. Se proponen principios y modelos educativos y se invita a los expertos a que aboguen por un modelo común e impulsen una red de formación europea.

Palabras clave: alcohol, educación médica, Europa, programas de formación.

\section{ABSTRACT}

The objective of this study is to review the current situation of medical education in Europe and to present some proposals for the future. With this aim, a thorough bibliographic review has been performed and several professionals and European universities have been contacted.

The collected information shows that medical education on alcohol is still a pending subject in Europe.

A basic training plan should cover the 3 levels of the medical career.

Key components (knowledge, skills and attitudes) and training strategies, mainly aimed at GPs and focussed on early identification and brief intervention are described.

Training objectives are: to know the facts, to identify alcohol problems, to distinguish whose patients may benefit from a brief intervention and to develop skills to apply it. Final goal should be the development of a therapeutic commitment. Educational principles and models are suggested and experts are invited to advocate for a common model and to strive for a European training network.

Key words: lalcohol, medical education, Europe, training programmes.

\section{INTRODUCCIÓN}

E consumo de alcohol comporta importantes problemas a nivel de toda la Región Europea. Los sistemas sanitarios podrían contribuir a prevenir y reducir el daño relacionado con el consumo de alcohol, si ampliaran su espectro de intervención a todos los bebedores. Los objetivos generales de salud para el año 2015 (HEALTH 21)' incluyen: "Proporcionar tratamiento asequible y eficaz a personas con consumo de riesgo o perjudicial, así como a aquellos que presentan una dependencia del alcohol". La consecución de este objetivo terapéutico implica una educación médica sólida y actualizada sobre el alcohol y sus efectos (presuntos beneficios, límites prudentes y de riesgo, problemas relacionados con el alcohol), así como sobre los diferentes abordajes terapéuticos. A la hora de ensanchar el espectro de intervención y asegurar una amplia disponibilidad de recursos, resulta clave la formación de los profesionales de la medicina,

\footnotetext{
* El presente artículo está basado en la ponencia presentada en la Conferencia Ministerial de la Organización Mundial de la Salud sobre Jóvenes y Alcohol (Estocolmo 19-21 de Febrero de 2001) dentro del grupo de trabajo Sistemas Sanitarios y Alcohol.
} 
especialmente de los médicos de atención primaria, a fin de que puedan identificar y abordar el consumo perjudicial o de riesgo.

\section{PESO DE LOS PROBLEMAS RELACIONADOS CON EL ALCOHOL EN EUROPA}

Se estima que el alcohol es responsable de aproximadamente el $10 \%$ de la morbilidad y del $50 \%$ de la mortalidad atribuible a lesiones en el conjunto de la Región Europea.

El nivel agregado de consumo alcohólico en la Unión Europea ${ }^{2}$ ha variado considerablemente como resultado de la homogeinización y de la globalización. El uso de las distintas bebidas se fue nivelando en los distintos países en los años 70 y ha continuado ${ }^{3}$, probablemente como resultado de una difusión transcultural. Sin embargo, los factores globalizadores no han impedido la supervivencia de diferentes culturas de consumo alcohólico, con patrones y niveles de bebida divergentes.

El reciente Estudio Comparativo Europeo sobre Alcohol (ECAS), patrocinado por la Comisión Europea y que incluye 14 países de la Comunidad Europea más Noruega, está generando interesantes resultados por lo que respecta a la evolución del consumo per cápita de alcohol y a la mortalidad por todas las causas entre 1950 y 1995. Parece existir una asociación entre el incremento del consumo global y el aumento de la mortalidad total, efecto que tiende a ser más acusado en los países de bajo consumo (nórdicos), pero donde abundan los episodios de embriaguez. En efecto, el peso cuantitativo y cualitativo de los problemas de alcohol parece reflejar tanto los niveles como las pautas de consumo.

El uso y abuso de alcohol es cada vez más prevalente entre los adolescentes europeos de 14 - 18 años. Los adolescentes de la Europa Occidental han incrementado la frecuencia de consumo, pero sobre todo los episodios de ingestión orientados a la embriaguez, fenómeno que aumenta con la edad, especialmente en los chicos. En Rusia ${ }^{4}$, se dan casos de psicosis alcohólica en adolescentes de 14 - 18 años (0.5/100.000 adolescentes en 1998). Alrededor de $12 / 100.000$ adolescentes han iniciado tratamiento en dispensarios de alcoholismo.

Los médicos necesitan tener amplia información sobre el consumo de bebidas alcohólicas y los problemas que del mismo se derivan (magnitud, tipo y distribución de los mismos). De esta forma podrán comprender la necesidad de identificar y aconsejar a sus pacientes con respecto a la cantidad y patrón de su consumo.

\section{AMPLIACIÓN DEL ESPECTRO DE INTERVENCIÓN Y DEL ESPECTRO DE TERAPEUTAS}

Teniendo en cuenta la distribución de los distintos tipos de bebedores y de las consecuencias del consumo a lo largo de un amplio espectro sin solución de continuidad, parece obligado ensanchar asimismo la base de la intervención. La prevención primaria, secundaria y terciaria deberían formar parte de un abanico de respuestas engarzadas ${ }^{5}$. De acuerdo con el concepto de la ampliación de la base del tratamiento y la hipótesis de la paradoja preventiva, los bebedores excesivos no dependientes deberían ser un objetivo diana para la promoción de salud y la prevención secundaria. A pesar de ciertas críticas vertidas sobre la paradoja preventiva, no hay duda de que la reducción de la ingestión de alcohol tiene un impacto favorable sobre los efectos agudos del alcohol.

El Plan Europeo sobre Alcohol para el periodo 2000- $2005^{8}$ subraya la necesidad de garantizar el tratamiento, no sólo a quienes padecen una dependencia alcohólica, sino también a todas las personas cuyo consumo de alcohol sea arriesgado o perjudicial. Los riesgos y problemas relacionados con el consumo de alcohol no pueden ser de la exclusiva competencia de los especialistas. En hospitales generales y escenarios sanitarios diversos debería poder realizarse el cribado y algún tipo de intervención. Ningún médico tiene derecho a pasar por alto problemas de alcohol pensando que "ya se encargará otro de ellos". Los bebedores excesivos y sus familiares hacen mayor uso de los servicios sanitarios y sociales que la población general. Los médicos tienen la posibilidad de comentar e intervenir sobre el consumo de sus pacientes porque éstos suelen considerarlos legitimados para ello.

Los profesionales sanitarios también deberían respaldar las políticas de alcohol y de promoción de salud, multiplicando de esta forma el espectro terapéutico.

\section{PAPEL DEL MÉDICO GENERALISTA}

Se considera que el médico de atención primaria se encuentra en una posición privilegiada para llevar a cabo una intervención precoz, por lo que debería ser 
formado para ello. En general, los médicos de cabecera son la puerta de entrada al sistema de salud y tienen la oportunidad de identificar a los pacientes con problemas de alcohol y decidir quién necesita ser derivado y quién puede beneficiarse de procedimientos menos específicos. La intervención breve suele ir dirigida a bebedores excesivos no dependientes y el cuestionario AUDIT fue desarrollado para permitir el cribado de bebedores de riesgo y con consumo perjudicial, especialmente en el marco de al atención primaria de salud.

\section{EDUCACIÓN MÉDICA EN EUROPA: UNA ASIGNA- TURA PENDIENTE}

El médico necesita una competencia basada en la adquisición de habilidades y unas actitudes positivas que le permitan enfrentarse debidamente a los problemas relacionados con el alcohol. Sin embargo, las facultades y escuelas profesionales no parecen proporcionar dicha formación. En Europa, la educación ha sido tradicionalmente escasa, con una media de 5-6 horas lectivas.

En 1989, la OMS publicó un informe sumario sobre el Simposio Europeo de Educación sobre Abuso de Drogas para Profesionales Sanitarios ${ }^{9}$ que incluía diversas recomendaciones:

- La formación en drogodependencias debería ser parte esencial de la formación médica, tanto a nivel curricular como de posgrado.

- Deberían ofrecerse cursos de posgrado

- La formación continuada debería ser ofrecida a los especialistas que ya trabajan en drogadicciones.

- La fórmula ideal sería la formación de formadores y los cursos deberían dirigirse a discípulos de múltiples profesiones.

- Periódicamente debería revisarse el estado de la cuestión a nivel nacional e internacional.

- Habría que promover el intercambio de información entre los países europeos. Esto crearía la base para una posible red formal de formación en drogodependencias a nivel europeo.

Al parecer, estas recomendaciones han sido sólo parcialmente seguidas. Todavía no existe en Europa ningún sistema estandarizado de formación de los médicos de atención primaria en la prevención e identificación precoz de problemas de alcohol y su correspondiente intervención. Ningún país posee un "programa modelo" que se extienda desde el pregrado al posgrado y sea implementado en todo el estado. No existe ningún programa nacional coordinado sobre drogodependencias ${ }^{10}$.

En conjunto, la educación sobre alcohol es escasa, fragmentada y repartida de forma deslavazada entre diversas asignaturas a lo largo de diferentes niveles de la carrera médica.

En la mayor parte de países europeos, las horas dedicadas a la educación de pregrado sobre alcohol son muy pocas. En general, las facultades de medicina se limitan a explicar el metabolismo del alcohol y las consecuencias físicas y psíquicas del consumo excesivo. Salvo honrosas excepciones, no suele existir tampoco educación sobre el contenido alcohólico de las bebidas ni sobre los limites del consumo prudente. Se dejan de lado la adicción y sus aspectos psicosociales.

Entre los estudios de posgrado, los estudiantes de psiquiatría y atención primaria tienen más probabilidades de abordar este tema, pero siempre prestando más atención a la patología inducida por el alcohol que a la dependencia alcohólica. Con excepción de algunos estudios reglados para obtener el título de "narcología" en la Europa del Este, la vía más habitual de familiarizarse con los problemas de alcohol son los cursos de máster o de experto en drogodependencias.

A pesar de la creciente formación a través de cursos de posgrado y de ciertos intentos de incluir el alcohol en el currículum de algunos países, todavía es posible graduarse como médico en Europa sin haber adquirido competencia alguna en adicciones ${ }^{10}$.

Las tablas 1 y 2 ofrecen una revisión panorámica, aunque no exhaustiva, que ilustra el estado de la cuestión y permite extraer un análisis hasta cierto punto particularizado. La dificultad en recopilar la información explica que no todos los países aparezcan en las tablas y que no todos los apartados puedan ser cumplimentados para cada país consignado. Más aún, al haber recopilado información de fuentes muy diversas, ha sido difícil estandarizarla, al tiempo que no se descarta algún pequeño error de interpretación por parte de la autora. No ha sido posible distribuir de forma precisa la educación médica entre pre- y posgrado debido, por una parte a la falta de información y, por otra, por la distinta consideración que merecen los diversos programas según los países y circunstancias. Por ejemplo, en España los cursos de posgrado son ofrecidos por la Universidad y los de formación continuada por instituciones no académicas. Un mismo "curso de máster" se considera como formación posgrado, cuando es presencial, y como formación continuada, si es suministrado como curso a distancia. 


\begin{tabular}{|c|c|c|c|}
\hline 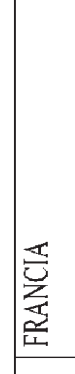 & 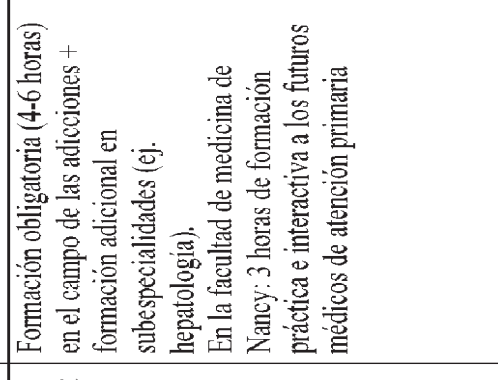 & 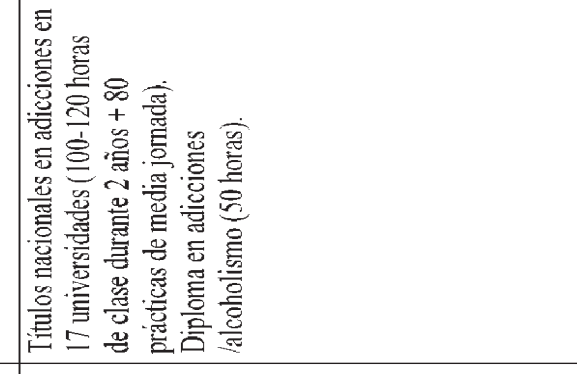 & 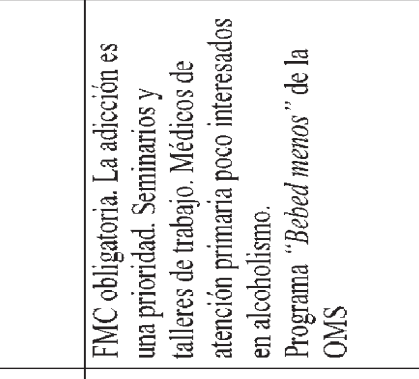 \\
\hline 亳 & 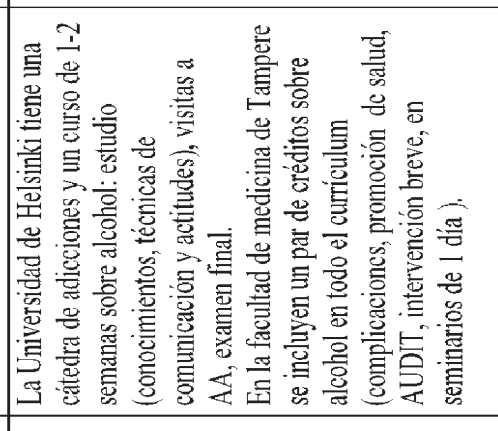 & 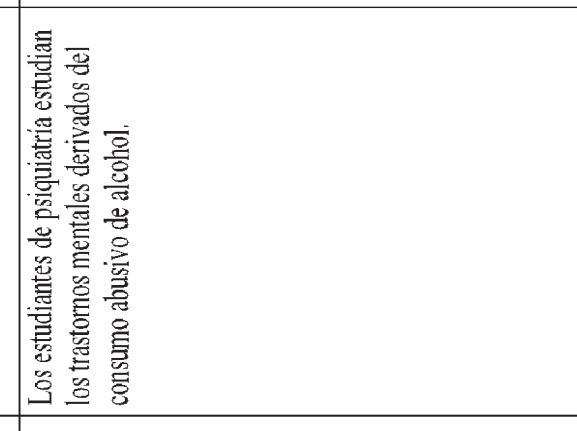 & 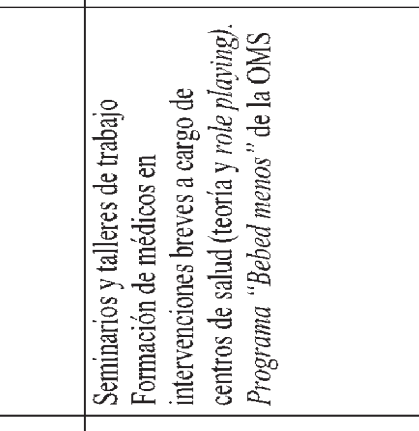 \\
\hline $\begin{array}{l}\text { 筤 } \\
\end{array}$ & 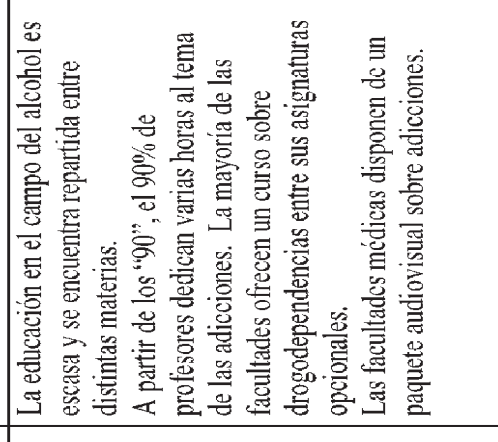 & 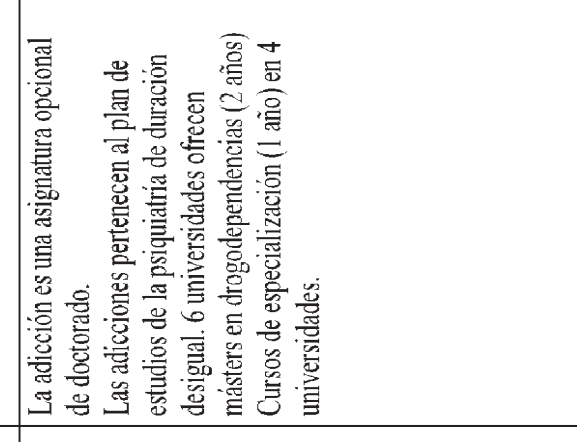 & 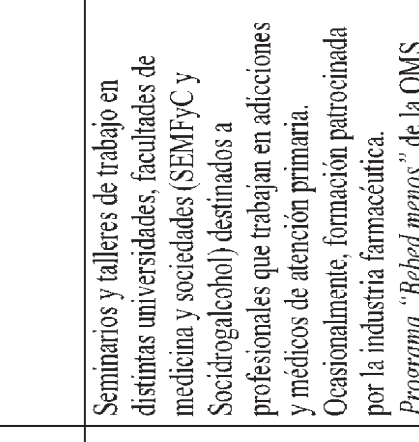 \\
\hline 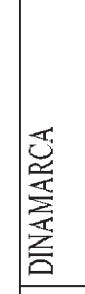 & 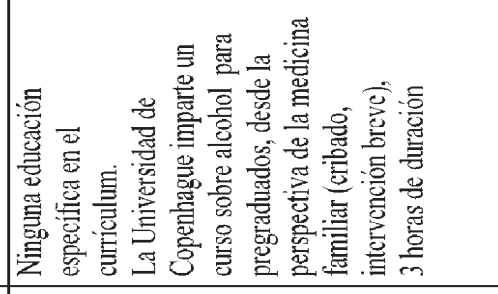 & 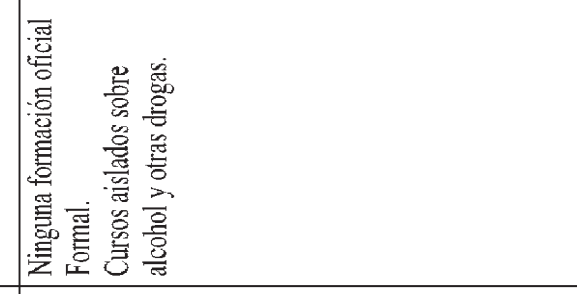 & 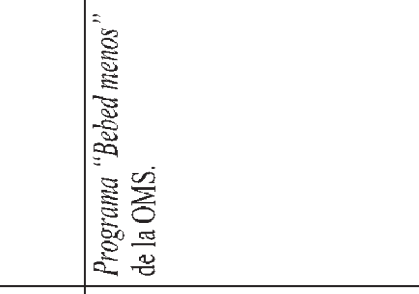 \\
\hline 浐 & 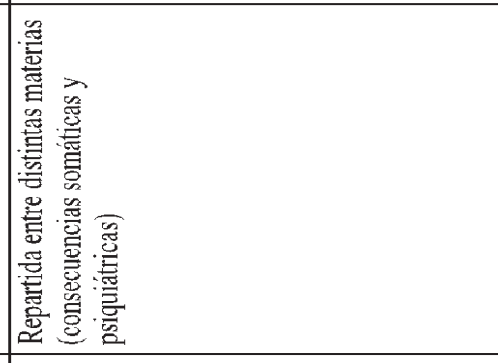 & 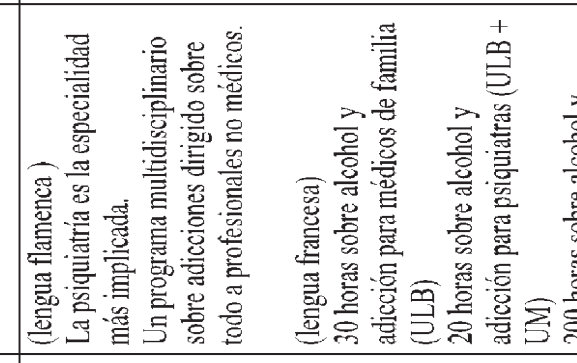 & 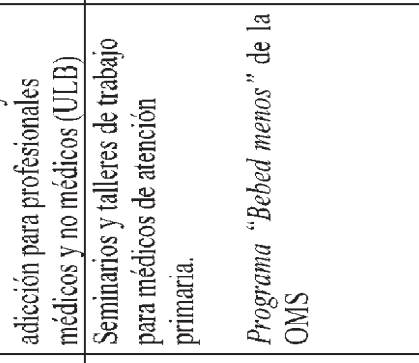 \\
\hline 童 & 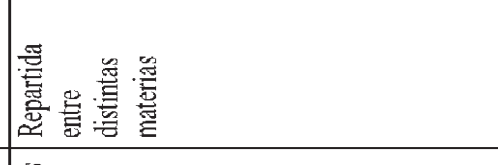 & 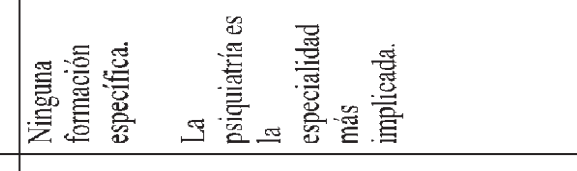 & 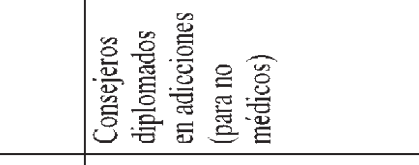 \\
\hline 慞 & 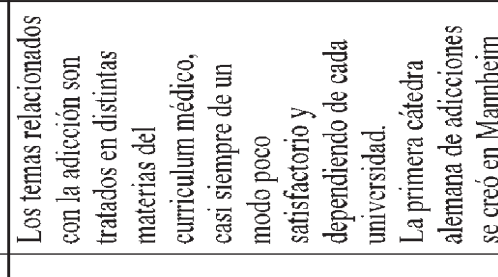 & 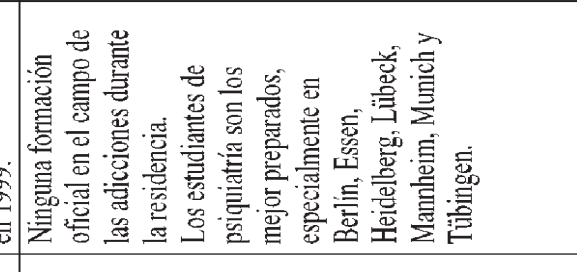 & 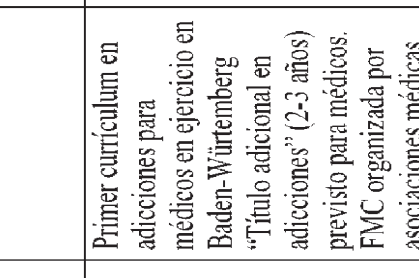 \\
\hline & 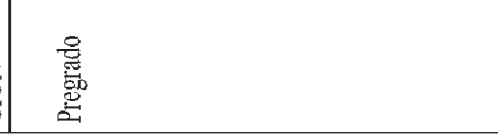 & 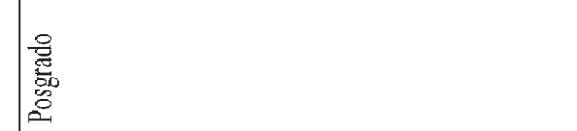 & 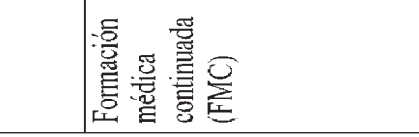 \\
\hline
\end{tabular}




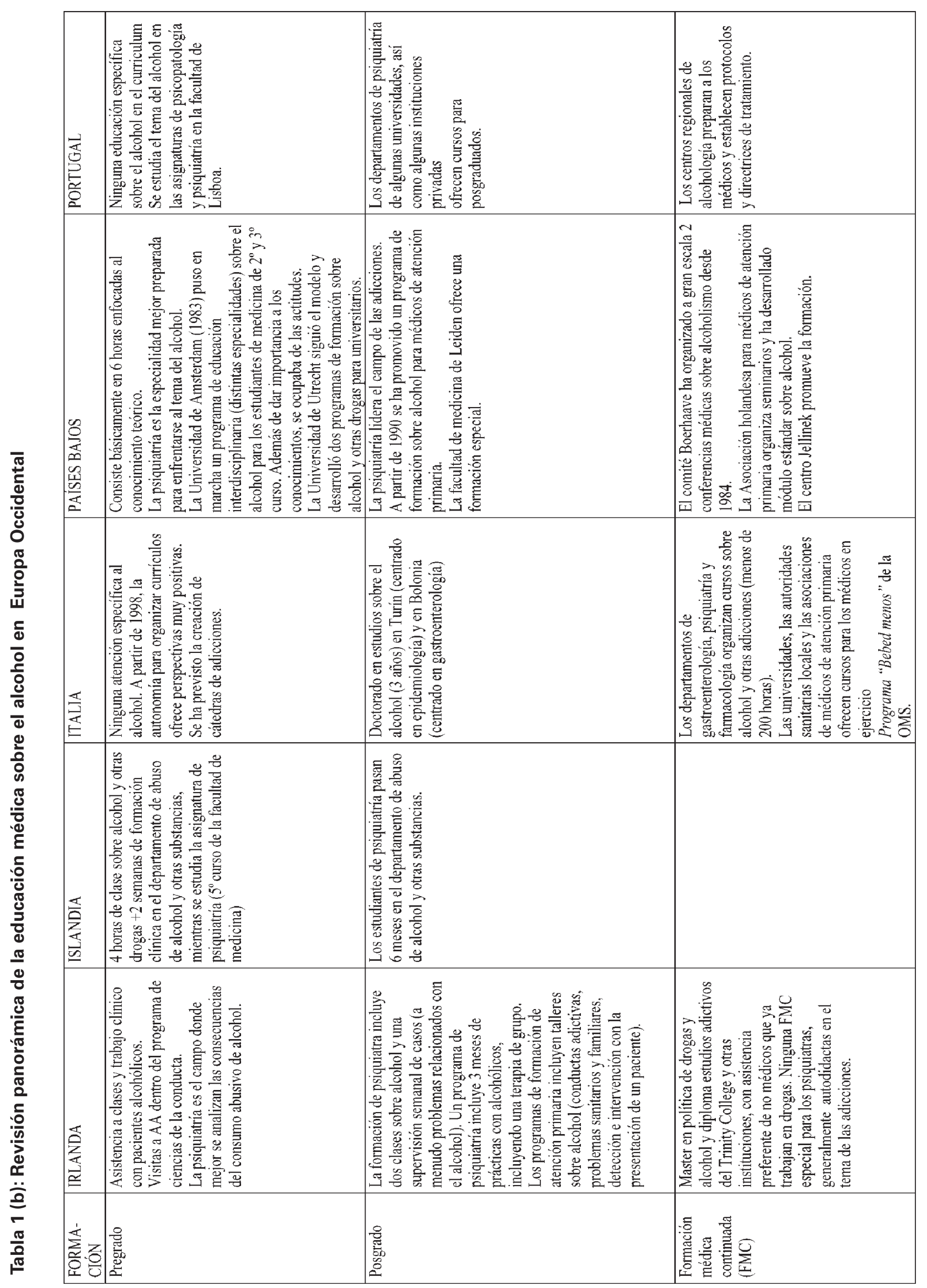




\begin{tabular}{|c|c|c|c|c|}
\hline $\mid \widehat{\Xi}$ & 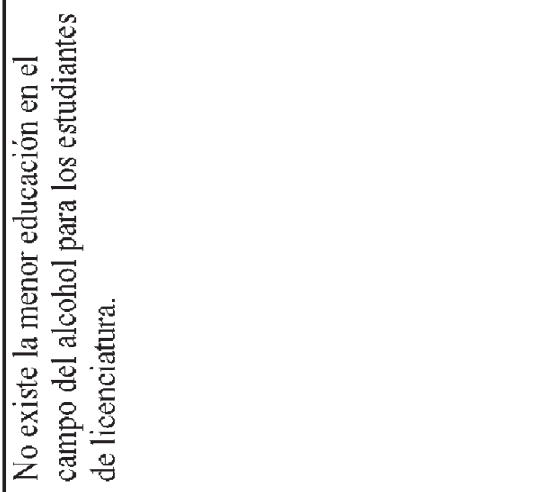 & & 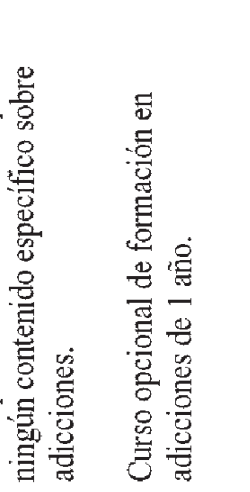 & 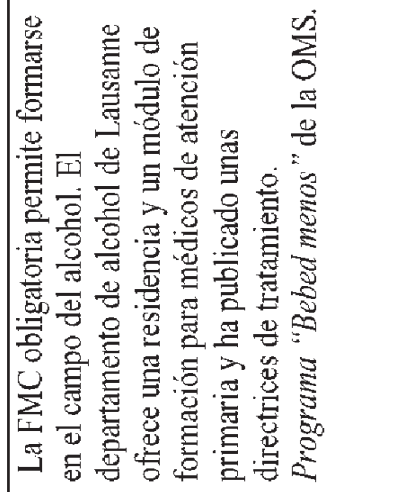 \\
\hline & 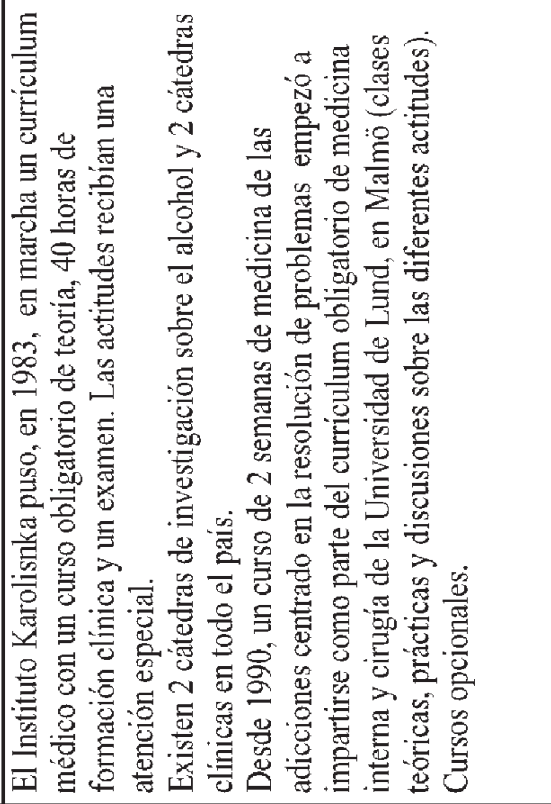 & 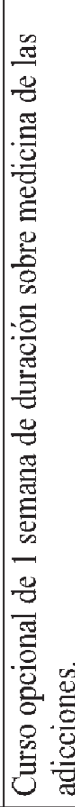 & 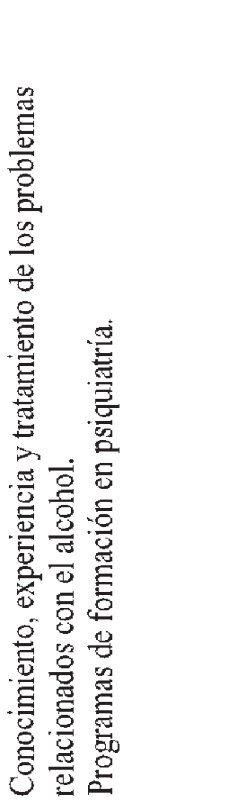 & 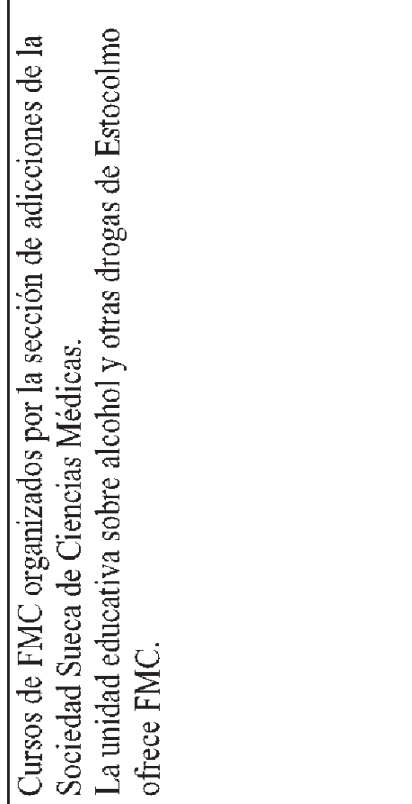 \\
\hline & 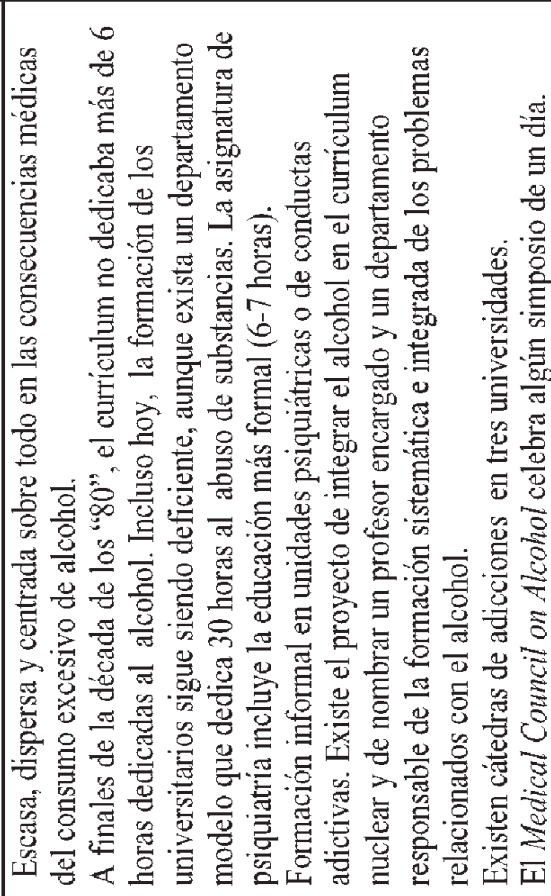 & 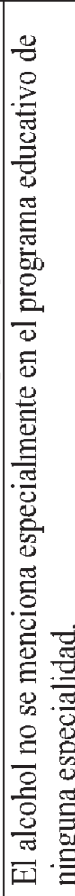 & 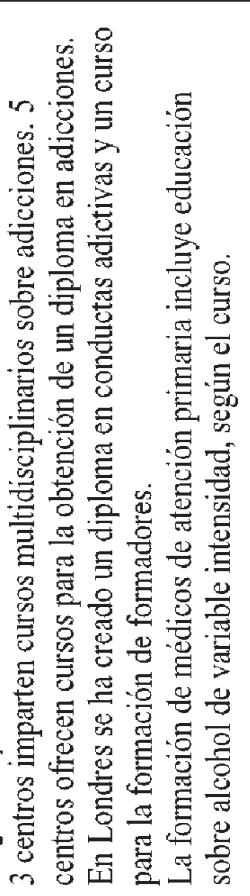 & 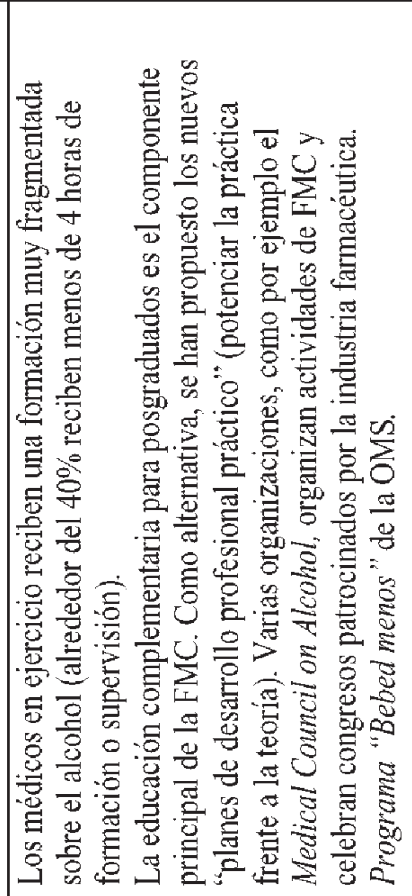 \\
\hline 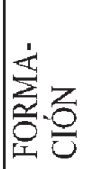 & 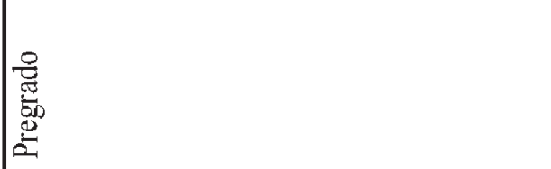 & $\begin{array}{l}\frac{\pi}{50} \\
0 \\
0 \\
0\end{array}$ & & 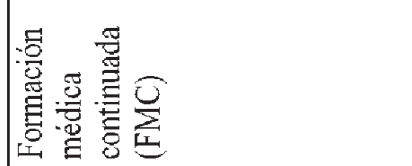 \\
\hline
\end{tabular}


En los países de Europa Occidental, la educación impartida sobre alcohol es todavía insuficiente, a pesar de los avances experimentados en estos últimos años..

La educación de pregrado sobre alcohol no suele pertenecer al currículum oficial. Se reparte de forma desigual entre las distintas materias (sobre todo en psiquiatría de pregrado), totaliza unas 6-8 horas y se centra en el metabolismo y consecuencias médicas del consumo de alcohol.

La educación de posgrado está muy extendida, alcanzando a veces un elevado nivel. Sin embargo, incluso los países con mejor educación sobre alcohol, suelen tener sólo una especie de educación "parcheada", con múltiples puntos flacos.

Las especialidades que dedican más tiempo a la formación sobre alcohol son la psiquiatría y la atención primaria. No existe una especialidad en adicciones. Incluso en países donde existen cátedras de conductas adictivas, éstas suelen limitarse a una universidad, lo que deja la educación sobre alcohol en manos del interés individual de los docentes.

Existen algunos títulos ("máster") en adicciones (en general con un programa de 2 años) en Francia, España e Irlanda. Italia posee un doctorado de 3 años en alcohol y, en España, las Adicciones son una asignatura opcional de doctorado. En Francia, Italia, España y Reino Unido existen diplomas (cursos de alrededor de 1 año) en conductas adictivas tanto en forma de posgrado como de formación médica continuada.

La formación médica continuada suele estar disponible en forma de seminarios, talleres, etc., pero no es obligatoria más que en contados países (Suiza y Francia). En ocasiones se forma a los médicos de atención primaria en intervención breve. 


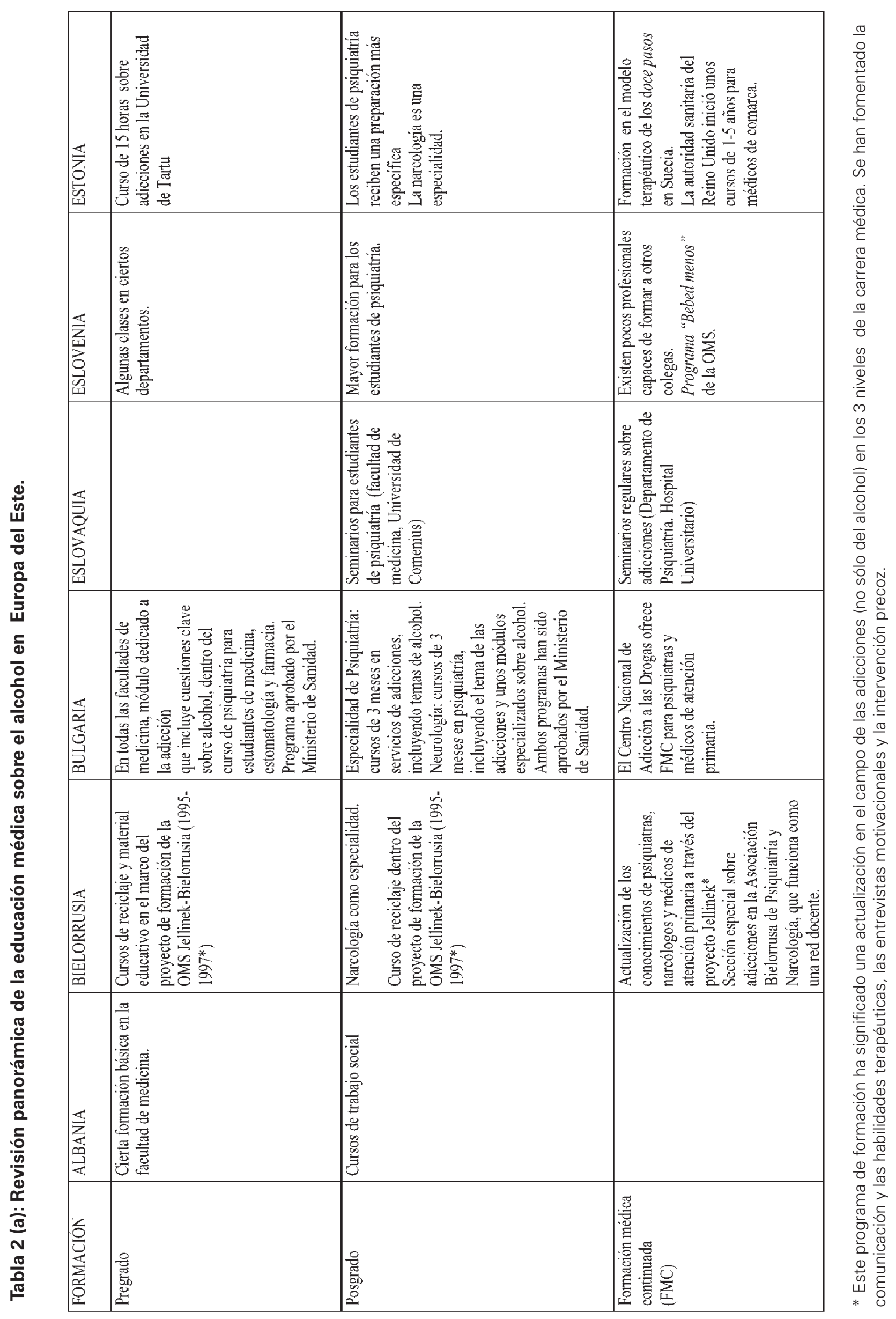



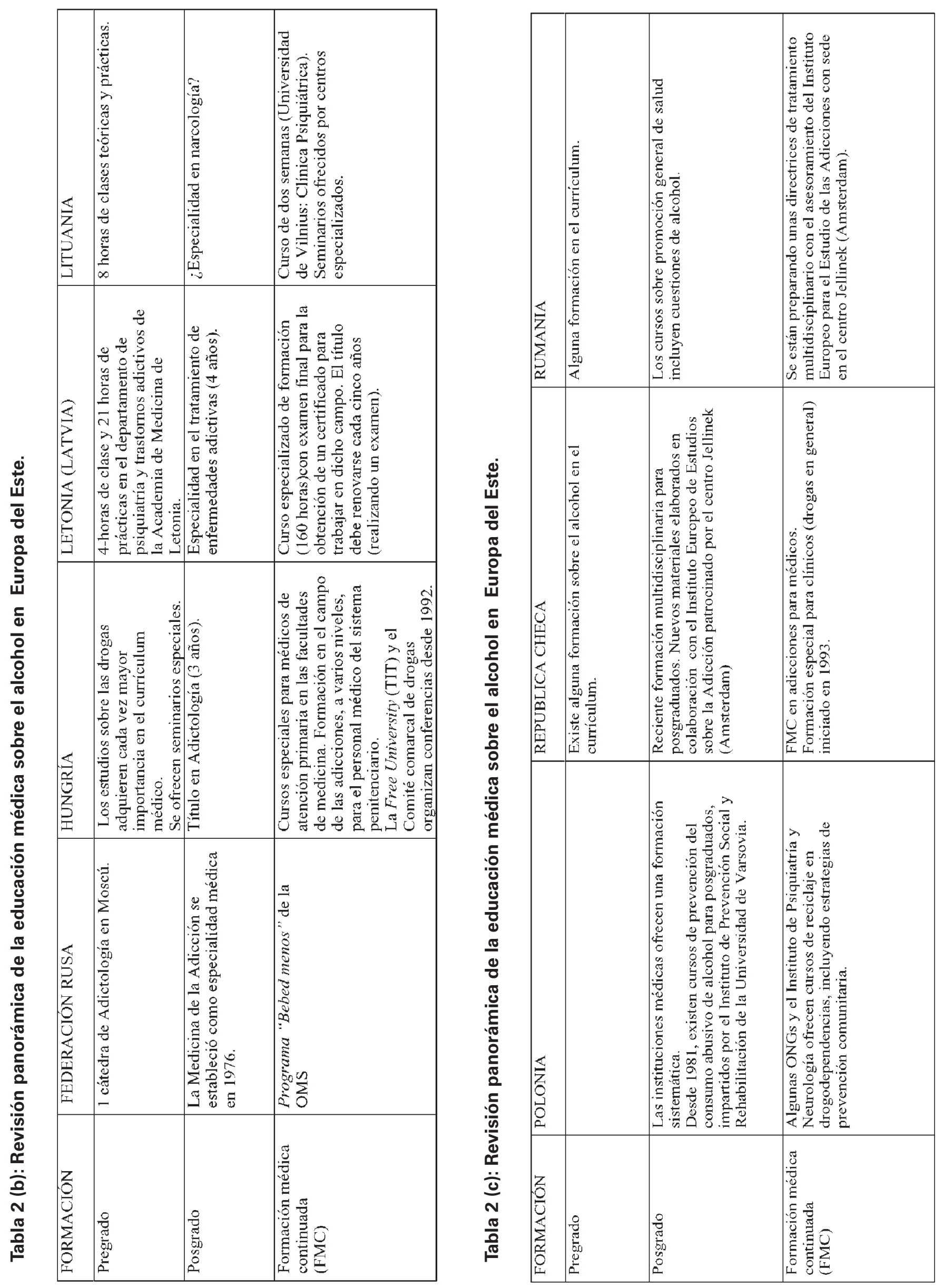
En Europa Oriental los médicos han estado tradicionalmente más familiarizados con el alcohol que con las drogas ilegales. En algunos países de la antigua Unión Soviética, existe la especialidad de "narcología." A los profesionales no especializados que trabajan en el campo de las adicciones se les pide al menos una cualificación en la materia a través de cursos diversos. No obstante, y a pesar de que existen diferencias notables entre un país y otro, en conjunto existe aún una formación insuficiente y no actualizada sobre alcohol en los currículos universitarios.

La formación de pregrado suele ser escasa (0-8 horas), aunque existen algunos programas amplios en países como Estonia (15 horas) y Letonia (4 horas de clase teórica y 21 de prácticas).

La educación de posgrado se ofrece en escuelas profesionales de psiquiatría, promoción para la salud y psicología médica. Las adicciones constituyen una especialidad médica en algunos países (ej.: Hungría, Latvia y Rusia).

La formación médica continuada (seminarios, etc.) se ofrece en algunos países, a veces con colaboración extranjera.

\section{TENDENCIAS FUTURAS...}

Los países europeos empiezan a ser conscientes de la carga que representan los problemas de alcohol y de la creciente conducta de riesgo frente al alcohol por parte de la juventud.12 países europeos han desarrollado un plan nacional sobre alcohol (Alemania, Estonia, Finlandia, Islandia, Israel, Irlanda, Italia, Lituania, Noruega, Países Bajos, Portugal, Suecia). 20 países (aquéllos con un plan nacional sobre alcohol más Bulgaria, Dinamarca, España, Georgia, Hungría, Luxemburgo, Malta, Rusia, Suiza y Urzbekistán) tienen una política nacional sobre alcohol y un organismo oficial coordinador. Alrededor de la mitad de ellos han definido algunos objetivos, la mayor parte relativos a la reducción del consumo per cápita.

\section{...Y NECESIDADES}

No obstante, para mejorar el papel del sistema sanitario en la reducción de daños relacionados con el alcohol, aún quedan muchas necesidades por cubrir.

Es necesario que los médicos abandonen mitos en torno a las virtudes del alcohol y al vicio del alcoholismo. Los profesionales han de tomar conciencia de sus propias incongruencias y su mentalidad de usuarios.
Los médicos se han de familiarizar con conceptos tales como consumo de riesgo, y ser conscientes de la elevada prevalencia de bebedores de riesgo entre sus pacientes, cuya evolución dependerá en gran manera de recibir un consejo de reducción a tiempo.

Se necesitan infraestructuras adecuadas, así como una buena formación de los profesionales, tema éste demasiado olvidado.

El encuentro sobre Educación Médica en Alcohol, celebrado en Lisboa en $1998^{10}$ subrayó la inadecuación de la formación actual y la necesidad de asumir un abordaje coordinado y multidisciplinario que garantizara una formación extendida a lo largo de toda la carrera profesional y basada en el conocimiento, las habilidades y las actitudes. Recogiendo las recomendaciones de la OMS, ${ }^{9}$ en el encuentro se animó a las autoridades sanitarias a que, en colaboración con los organismos nacionales e internacionales, impulsaran la implementación de una formación global, abarcando desde la etapa pregrado hasta la educación médica continuada, y el desarrollo de un sistema de calificación acorde con estándares homogéneos.

El Plan Europeo sobre Alcohol ${ }^{8}$ subraya también la necesidad de "facilitar la formación de los profesionales de atención primaria para que puedan identificar a los bebedores con consumo perjudicial o de riesgo e intervenir sobre ellos y la intervención sobre ellos".

Teniendo en cuenta todos estos precedentes, esta ponencia propone una educación básica sobre alcohol orientada a la prevención y la reducción del daño en toda la Región Europea.

\section{PROPUESTAS PARA UN PROGRAMA DE EDUCA- CIÓN BÁSICO SOBRE EL ALCOHOL Y LOS PRO- BLEMAS RELACIONADOS CON SU CONSUMO}

Los profesionales han de adquirir criterios científicos libres de prejuicios culturales, y habilidades médicas y de comunicación personal. La formación sobre alcohol ha de estar distribuida en los 3 niveles educativos:

Educación de pregrado: el currículum médico debería incluir siempre una educación "nuclear" en temas de alcohol que permitiera un ejercicio de la profesión de orientación comunitaria"1. El British Medical Council on Alcoholism (MCA) propone 8 temas objetivo de aprendizaje: 1) alcohol; 2) alcohol e individuo; 3) coste del abuso alcohólico; 4) problemas clínicos; 5) implicaciones psiquiátricas; 6) identificación y reconocimiento; 7) tratamiento y 8) políticas. Otro tema que podría añadirse es el de alcohol y familia. En cada facultad de medicina, un departamento debería orga- 
nizar la cobertura sistemática de estos temas y un profesor designado podría ser el responsable de la educación sobre alcohol, con la colaboración y el consenso de diferentes cátedras ${ }^{11}$, cuando no exista una cátedra de conductas adictivas, como suele ser el caso. Habría que promocionar y apoyar el desarrollo y supervivencia de las unidades de conductas adictivas donde las haya.

Estudios de posgrado: todo médico debería disponer de una oferta de cursos de posgrado. La formación en alcohol debería ser especialmente concienzuda en algunas especialidades, como psiquiatría y atención primaria, cuyo ejercicio requiere una amplia y profunda formación en estos temas.

La formación médica continuada debería actualizar los conocimientos de los médicos en ejercicio, así como servir para cubrir los posibles huecos en la formación previa. Se recomiendan encarecidamente el aprendizaje multidisciplinario y la formación basada en la práctica. Además de las tareas docentes propiamente dichas, la formación continuada ha de considerar los factores predisponentes, facilitadores y de refuerzo.

\section{PROGRAMAS DE FORMACIÓN PARA MÉDICOS DE ATENCIÓN PRIMARIA}

En 1992, un grupo de trabajo de la OMS ${ }^{12}$ identificó 12 competencias requeridas por los médicos, sobre todo de atención primaria, para el correcto abordaje (prevención y tratamiento) de los problemas de alcohol. Son éstas:

1. Conocimiento de la prevalencia del consumo peli groso y perjudicial de alcohol y de los problemas físicos, psíquicos y sociales con él relacionados.

2. Conocimiento y apreciación de los efectos del problema alcohólico del paciente en su pareja o familiares.

3. Conciencia de la actitudes personales del paciente respecto al alcohol.

4. Habilidad para identificar los diversos indicadores de problemas físicos, psicológicos y sociales relacionados con el alcohol.

5. Habilidad para comunicar la información sobre el alcohol y los problemas relacionados con su consumo a los pacientes y sus familiares, en el contexto adecuado.

6. Habilidad para distinguir entre los niveles de consumo de riesgo bajo, peligroso o, perjudicial y el consumo dependiente.
7. Habilidad para tratar las consecuencias físicas y las complicaciones de la intoxicación aguda.

8. Habilidad para recoger una historia clínica precisa.

9. Habilidad para reconocer los signos de una enfermedad relacionada con el alcohol.

10. Habilidad para interpretar los marcadores biológicos de forma adecuada.

11. Habilidad para elegir el plan terapéutico más adecuado (intervención breve o derivación a los colegas o servicios adecuados).

12. Habilidad para dirigir y manejar la desintoxicación domiciliaria.

Teniendo en cuenta estas recomendaciones y la actual promoción de la identificación precoz e intervención breve, los programas de formación para médicos de atención primaria deberían orientarse a la consecución de 4 objetivos nucleares de aprendizaje y una meta de formación final.

Objetivos nucleares de aprendizaje:

- Conocer los hechos y conceptos.

- Ser capaz de hacer un cribado de los problemas de alcohol.

- Ser capaz de distinguir qué pacientes necesitan y cuáles pueden beneficiarse de una intervención breve y cuáles requieren una derivación a programa terapéutico.

- Desarrollar habilidades para comunicar la información a los pacientes y suministrarles un consejo breve.

La meta terapéutica final es que el médico tome conciencia de su papel en los problemas de alcohol y desarrolle el correspondiente compromiso terapéutico.

A fin de alcanzar dichos objetivos, los programas de formación en identificación precoz e intervención breve deberían estar basados en 3 componentes clave (conocimientos, habilidades y actitudes) y la utilización de las adecuadas estrategias docentes.

\section{A. Componentes clave}

\section{Conocimientos}

1 a. Conocimientos sobre los hechos

- Criterios sobre consumo de riesgo, consumo perjudicial y dependencia alcohólica (clasificación CIE-10). 
- Espectro de bebedores (distribución de los bebedores a lo largo de un continuum de consumidores y niveles de riesgo).

- Problemas físicos, psicológicos y sociales relacionados con el alcohol (morbilidad y mortalidad).

- Dimensión del riesgo: balance entre los pretendidos beneficios (mitos culturales y conocimientos basados en la evidencia) y daños causados por el alcohol.

1 b. Conocimientos sobre el cribado.

- Cribado del consumo excesivo: cómo y cuándo preguntar sobre el consumo de alcohol (concepto de bebida estándar).

- Cribado de los problemas relacionados con el alcohol: cuándo y cómo emplear e interpretar cuestionarios diagnósticos (por ej.: AUDIT, etc.) y marcadores biológicos.

- Indicadores de consumo perjudicial.

1 c. Conocimientos sobre intervenciones breve.

- Concepto de la intervención breve y su substrato teórico.

- Modelo del cambio de Prochaska y DiClemente.

- Fundamentos del abordaje motivacional.

- Elementos activos de la intervención breve (acrónimo FRAMES).

- Beneficios de la intervención breve (resultados de la investigación sobre el tema).

- Papel de la atención primaria de salud.

- Técnicas de intervención breve: consejo simple frente a intervención breve motivacional.

- Indicaciones respectivas de intervención breve y derivación.

- Desintoxicación domiciliaria (indicaciones y estrategias).

1 d. Otros conocimientos útiles y complementarios

- Coste de los problemas de alcohol.

- Conocimientos básicos sobre los programas terapéuticos (deshabituación).

- Conocimientos básicos sobre el tratamiento farmacológico de la dependencia alcohólica (indicaciones, efectos secundarios e interacciones terapéuticas).

- Conocimientos básicos sobre resultados del tratamiento y recaídas.

- Información sobre material de apoyo para pacientes (folletos de autoayuda) y para los propios médicos (directrices, paquetes de programas y listado de recursos locales para la derivación de casos).

\section{Habilidades}

- Habilidades para detectar y valorar los efectos del abuso de alcohol en el paciente y su familia.

- Habilidades para comunicar la información sobre los riesgos y daños producidos por el alcohol a pacientes y familiares.

- Habilidades para recoger una buena historia clínica.

- Habilidades para aproximarse al paciente sin juicios morales, con un estilo empático y motivacional.

- Habilidades para elegir el mejor abordaje (intervención breve o derivación) para el paciente, teniendo en cuenta sus necesidades y actitudes.

- Habilidades para el consejo.

- Habilidades para alcanzar con el paciente un mutuo compromiso terapéutico.

\section{Actitudes}

- Actitudes exentas de juicios de valor (ser consciente de los propios prejuicios y de la mentalidad de usuario).

- Respeto y empatía.

- Expectativas equilibradas.

- Confianza en sí mismo.

\section{B. Estrategias}

Las estrategias para los programas de formación han de incluir la transmisión de conocimientos teóricos, el adiestramiento en habilidades prácticas y la promoción de actitudes positivas, así como de un compromiso terapéutico. Es altamente recomendable incluir un examen final.

\section{Estrategias para la transmisión del conocimiento teórico}

La transmisión de conocimiento teórico debe hacerse por medio de clases didácticas, seminarios y bibliografía recomendada.

Hay que trabajar a fondo el modelo del cambio de Prochaska y DiClemente e introducir los conceptos 
básicos de la entrevista motivacional como fundamento teórico para la intervención breve.

Es importante facilitar materiales de apoyo tales como cuestionarios y otros instrumentos diagnósticos (por ej.: tabla con el contenido alcohólico de las distintas bebidas y equivalencia en unidades de bebida estándar), directrices, diagramas, dípticos para pacientes y listado de centros para derivación.

Las sesiones han de ser interactivas para garantizar que los conceptos expuestos han sido bien entendidos. La información debe centrarse en los conocimientos clave y limitarse a hechos contrastados. No debe perderse el tiempo de las sesiones aportando conocimientos de alta especialización o información no relevante. Los grupos de formación no deben exceder las 30 personas que, en lo posible, han de pertenecer al mismo grupo profesional (por ej.: médicos de atención primaria) o ser profesionales diversos trabajando conjuntamente en equipos multidisciplinarios. De este modo, se dará respuesta a las necesidades al tiempo que se eliminan barreras.

Es importante que las sesiones sean breves, aunque formen parte de cursos de formación intensiva.

\section{Estrategias para el entrenamiento en habilidades}

El mejor modo de desarrollar habilidades y adquirir confianza en sí mismo es el entrenamiento práctico. Una formación ideal debe incluir algunos ejercicios prácticos, role playing (juego de roles) y estudio de casos ( si es posible, prácticas con pacientes reales)

Los ejercicios prácticos deberían incluir:

- Cribado de problemas de alcohol

- Cálculo del consumo de alcohol

- Aplicación e interpretación de cuestionarios tales como AUDIT, CAGE...

- Interpretación de marcadores biológicos

- Identificación del estadio de cambio

- Estudio de casos (caso teórico discutido en grupo)

- Clasificación de los trastornos relacionados con el alcohol según la CIE-10

- Intervención (consejo breve)

- Role playing en intervención breve (simple consejo e intervención breve de tipo motivacional) teniendo en cuenta las posibles circunstancias, edad y sexo del paciente

- Role playing en intervenciones familiares
- Discusión sobre resistencias y estrategias facilitadoras observadas en el curso del role playing o en las cintas de vídeo analizadas

Los estudiantes deben aprender a integrar la intervención breve dentro del proceso secuencial iniciado por la identificación precoz y evaluación. El entrenamiento práctico es sinónimo de supervisión (retroalimentación y apoyo del rol).

3. Estrategias para la promoción de actitudes positivas y del compromiso terapéutico

Las actitudes no pueden ser enseñadas, pero sí promovidas. Las actitudes dependen del conocimiento, pero también de las emociones y de la intención de conducta. Los patrones culturales y los prejuicios sociales ejercen una influencia sobre la actitud de los profesionales y deben ser discutidos. A su vez, la conducta personal y profesional modifica las actitudes. He aquí algunas estrategias útiles:

- Discusión y análisis de las actitudes personales ...

1. Con respecto a la bebida y la conducta consumidora

2. Generadas al entrevistar al paciente (barreras, factores facilitadores, confianza en sí mismo, expectativas...)

3. Generadas durante el role playing (rol de terapeuta o paciente).

- Discusión sobre el compromiso terapéutico

La confianza en uno mismo (seguridad de rol) promueve el compromiso terapéutico, cuyos 3 factores facilitadores ${ }^{13}$ cabe discutir en grupo

1. Adecuación del rol, que depende de las habilidades desarrolladas y de la dificultad de los casos previamente tratados (durante la formación, la exposición a pacientes con problemas recientes debería superar a la correspondiente a casos de larga evolución).

2. Legitimidad del rol, que puede ser descrita, pero que se desarrollará especialmente con la práctica clínica.

3. Soporte del rol (supervisión y refuerzo), que es el elemento clave del compromiso terapéutico.

Los programas de formación deben proporcionar una competencia basada en las habilidades, autoeficacia y expectativas realistas, que son a su vez el principal determinante de una práctica exitosa ${ }^{14}$ y refuerzan el compromiso terapéutico. 
La figura 1 ilustra la interrelación de factores que concurren en el desarrollo de las actitudes positivas y el compromiso terapéutico.

\section{Figura 1. Círculo de actitudes positivas y compromiso terapéutico}

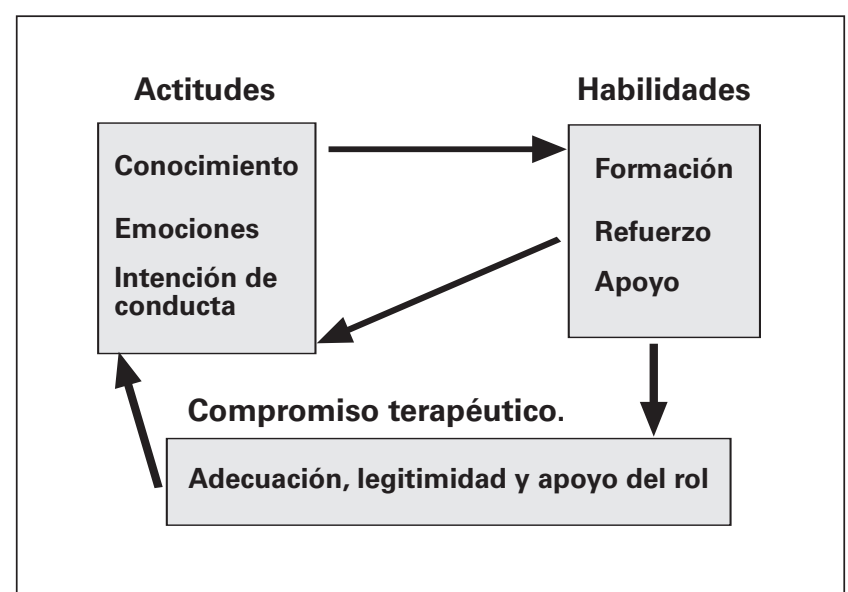

\section{PRINCIPIOS EDUCATIVOS DE LA FORMACIÓN POSGRADO}

Una buena formación de posgrado requiere, además de los componentes básicos de los programas antes citados, la incorporación de algunos principios educativos ${ }^{5}$ :

Estrategias centradas en el discípulo: se trata de trabajar conjuntamente con los alumnos a fin de seleccionar los objetivos docentes adecuados a sus necesidades.

Dichas necesidades pueden ser identificadas por un focus group ${ }^{15}$ (grupo seleccionado), que debería incluir a colegios y sociedades médicas junto con expertos. Los paquetes de formación y estrategias deben de adaptarse a las necesidades y circunstancias de trabajo de los discípulos. Los materiales de apoyo y las directrices para la intervención han de adecuarse a las posibilidades reales de su implementación. Los cursos han de ser compatibles con el horario laboral de los médicos.

Estudio de casos: la discusión de casos con los alumnos potencia sus actitudes positivas.

Entrenamiento basado en la experiencia: incluye la discusión sobre experiencias personales, juegos de rol y dinámica de grupos. Dichas estrategias permiten analizar los sentimientos personales y actitudes, así como desarrollar habilidades prácticas de intervención.

Experiencia longitudinal: incluye la implicación de los estudiantes antes, durante y después del curso (envío de literatura o una lista de lecturas recomenda- das antes de empezar el curso; diseño de un plan específico para implementar lo que ha aprendido, el alumno; seguimiento y apoyo (mediante llamadas telefónicas o entrevistas) de los formados por parte de sus formadores.

Vínculos: proporcionar una lista actualizada de los recursos terapéuticos locales para derivar al paciente o colaborar en el caso. También hay que suministrar conexiones para facilitar el ulterior asesoramiento y refuerzo. Para lograr una amplia implementación y difusión de la identificación precoz e intervención breve, hay que establecer alianzas estratégicas con autoridades académicas, así como con colegios y sociedades profesionales.

Cambio de rutinas terapéuticas: se trata de ayudar a los discípulos a descubrir prácticas que pueden ser modificadas o mejoradas en aras a una mejor detección e intervención terapéutica.

\section{MODELOS PARA LA FORMACIÓN MÉDICA CONTI- NUADA}

Aunque la formación médica continuada no es aún obligatoria, para algunos países es todavía la única manera de adquirir conocimientos sobre alcohol o de actualizarlos. Existe cierta experiencia en cursos a distancia que incluyen evaluación y créditos académicos.

Anderson ${ }^{5}$ sugiere los siguientes modelos de educación médica continuada:

Programas de formación de formadores: pueden ser administrados como educación médica continuada por parte de instituciones diversas (facultades de medicina, escuelas profesionales, colegios médicos, sociedades de medicina familiar o atención primaria y de adicciones, etc.).

La formación de formadores permite abarcar un espectro más amplio de médicos de atención primaria a través de un efecto multiplicador. Los alumnos pueden ser médicos de atención primaria o médicos que trabajen en el campo de las adicciones. El modelo de formación de formadores es una buena alternativa. El desarrollo del modelo de formación estará, en cualquier caso, en función del programa de intervención a aplicar.

Consulta in situ: consiste en disponer de un facilitador que asesore periódicamente a la plantilla de atención primaria.

Abordaje sistémico: pretende ayudar al médico de atención primaria a ampliar su visión de la promoción de salud y desarrollar estándares de buena práctica profesional. 
Cursos de formación para la promoción del cambio: se basan en el modelo del cambio y el abordaje motivacional y enseñan el modo de ayudar a cambiar a los pacientes.

La formación médica continuada permite la difusión del cribado y la intervención breve. A su vez, algunos proyectos de investigación promueven la formación y el compromiso personal en la intervención precoz. Entre sus estrategias para la diseminación de la identificación precoz e intervención breve, el Proyecto de Colaboración de la $\mathrm{OMS}^{15}$ incluye las siguientes actuaciones:

- Crear materiales y servicios a medida del destinatario

- Relectura de la cuestión del alcohol

- Establecer organizaciones líder y construir alianzas estratégicas

- Establecer y evaluar ensayos.

\section{CONCLUSIÓN}

La perspectiva futura es positiva, aunque queda todavía mucho camino por recorrer y se precisan revisiones periódicas sobre el estado de la cuestión?

Teniendo en cuenta los objetivos generales de salud para el año 2015 (HEALTH 21 1), la formación de los médicos de atención primaria en identificación precoz e intervención breve es indispensable. Sin embargo, no cabe olvidar a otros profesionales de la medicina. Todo médico debería ser consciente de los riesgos y daños producidos por el alcohol y asumir el compromiso de intervenir sobre ellos. El cumplimiento de esas expectativas exige una mejor formación más amplia, y coordinada, así como un abordaje más plural. Los expertos y organismos europeos deberían acordar un modelo educativo y su adecuado sistema de calificación. Deberían abogar por un cambio curricular que asegurara una formación integral, extendida a lo largo de los 3 niveles de la carrera médica, así como velar por su implementación, sentando así las bases para una red de formación europea.

\section{BIBLIOGRAFÍA}

1. World Health Organization: Health 21: The health for all policy framework for the WHO European Region, Copenhagen. WHO Regional Office for Europe, Copenhagen 1999 (European Health for All Series No.6)

2. Leifman, $\mathrm{H}$.: Trends in Alcohol Consumption in the Euroean Union. Paper presented at 26th Annual
Alcohol Epidemiology Symposium of the Kettil Bruun Society, Oslo, Norway, 5-9 June 2000.

3. Holder $\mathrm{H}$ et a.: European integration and Nordic alcohol policies. Eds. Holder H, Kühlhorn E, Nordlund $S$, Österberg E, Romelsjö A, Ugland T. Adershot (England), Ashgate, 1998.

4. Koshkina E; Vyshinsky K. Medical and social consequences of alcohol abuse in Russian federation according to official statistical data. Paper presented at the 26th Annual Alcohol Epidemiology Symposium of the Kettil Bruun Society, Oslo, Norway, 5-9 June 2000.

5. Anderson P. Alcohol and primary health care. Who Regional Publications. European Series N 64. Geneva, WHO 1996.

6. Institute of Medicine. Broadening the base of treatment for alcohol problems. Washington DC, National Academy Press, 1990.

7. Haether N. Where treatment merge: the need for a broader approach. Addiction 1997 (suppl. 1): S 133S136.

8. World Health Organization: European Alcohol Action Plan 2000-2005: WHO Regional Office for Europe, Copenhagen 1999 (EUR/RC49/9).

9. World Health Organization: Summary Report of the European Symposium on Substance Abuse Education for Health Professionals. The Hague, 9-10 October 1989, 3 pp.

10. Medical Education in Alcohol and Alcohol Problems: A European Perspective. EUROCARE 1999, 46 pp.

11. Mc Avoy, B. Alcohol-education for general practitioners in the United Kingdom - A window of opportunity? Alcohol \& Alcoholism 2000; 35 (3): 225-229.

12. World Health Organization: The role of general practice settings in the prevention and management of the harm done by alcohol use. Report on a WHO meeting. Copenhagen, WHO Regional Office for Europe, 1992 (EUR/ICP/ADA 038).

13. Shaw et al.: Responding to Drinking Problems. London, Crome Helm, 1978.

14. Saunders, JB; Roche, A.M: Medical education in substance use disorders. Drug and Alcohol Review 1991; 10: 263-275.

15. WHO Collaborative Project on Identification and Management of Alcohol-Related Problems in Primary Health Care (Phase IV) Study Protocol version 04/05/99.

\section{AGRADECIMIENTOS}

Desearía expresar mi agradecimiento a muchos colegas de diferentes países europeos por su amable colaboración y la valiosa información suministrada. 
